\title{
Los diseños motivacionales en ambientes virtuales de aprendizaje en la enseñanza del inglés
}

\section{Motivational designs in virtual learning environments in English teaching}

\author{
Edgar Ramírez Ramírez \\ edgarramirezramirez@gmail.com \\ Código ORCID: 0000000308808939 \\ Institución Educativa Agustín Nieto Caballero, Colombia
}

- Artículo recibido en abril 2020

RESUMEN

- Publicado en julio 2020

El aprendizaje de inglés es considerado como un complemento del currículo y muchas de sus prácticas son sinónimo de monotonía, repetición y, por lo tanto, de poca motivación. Los ambientes virtuales de aprendizaje pueden mediar muchos tipos de saberes, para lo cual su utilización debe obedecer a una planificación cuidadosa, con ello cada una de las etapas de la clase contará con elementos motivadores que generen entusiasmo, y potencialicen la adquisición y desarrollo de habilidades de la lengua extranjera. Al combinar saberes de la psicología, la pedagogía y la didáctica se ha creado un diseño motivacional con el apoyo de las TIC para estudiantes de educación básica. El producto, en formato de vídeo juego, indaga por las sensaciones y conocimientos de los sujetos que intervienen y se proyecta para continuar profundizando en los procesos de enseñanza y aprendizaje a través de las secuencias didácticas mediadas por las experiencias virtuales.

Palabras clave: Enseñanza del inglés; TIC; motivación; vídeo juego

\section{ABSTRACT}

Learning English is considered as a complement to the curriculum, and many of its practices are synonymous with monotony, repetition, and therefore little motivation. Virtual learning environments can mediate the study of this and other knowledges, for which its use must obey to a careful planning for each of the stages of the class with motivating elements that generate enthusiasm, and enhance the acquisition and skills development of the foreign language. By combining knowledge of psychology, pedagogy and didactics, a motivational design has been produced with the support of ICT for basic education students. The product in video game format researches the sensations and knowledge of the involved subjects and it is projected to continue into the deepening of the teaching and learning processes through didactic sequences mediated by virtual experiences.

Keywords: Teaching English; ICT; motivation; video game 


\section{INTRODUCCIÓN}

Se presenta una aproximación al estudio de las mediaciones de las Tecnologías de la Información y la Comunicación (TIC) para llevar a cabo con éxito los procesos de enseñanza y de aprendizaje de una segunda lengua. Se describe cómo las TIC cumplen un papel motivador en la enseñanza del inglés en una escuela pública de la ciudad de Cali, Colombia. El trabajo abarcó los temas de la motivación en educación, la enseñanza del inglés, los ambientes virtuales de aprendizaje y la gamificación.

En esta oportunidad se muestran elementos vinculados a los ambientes virtuales de aprendizaje y sus primeras experiencias de aplicación para lograr el aprendizaje del inglés, en una propuesta lúdica y motivacional de carácter interactivo.

Sobre la motivación a aplicar en educación es conveniente potenciar la intrínseca, la cual puede generar autodeterminación para algunos autores o autonomía según otros (Orbegoso, 2016; Santos Marín, 2017). Sin embargo, no se descarta del todo que la motivación extrínseca se pueda convertir en intrínseca en muchos de los procesos de aprendizaje (Camposeco, 2012), especialmente en un trabajo con el que los estudiantes se relacionan estrechamente y se sienten promotores de su propia ilustración. Se encontró, igualmente que, en la enseñanza de inglés, al igual que en todas las otras áreas del conocimiento, la motivación juega un papel fundamental al momento de lograr un aprendizaje permanente, disciplinado y a largo plazo. Cuando determinadas didácticas y estrategias son aplicadas para lograr un interés extra del estudiante, muchas de esas mediaciones tienen incluidas el aprendizaje, utilizando las TIC.

Pero estos programas, desde hace ya algunos años atrás, son aplicaciones que han dejado de ser consideradas solo herramientas, y se han convertido en unos verdaderos transformadores, mediadores de aprendizaje (Anaya, Díaz y Martínez, 2012; Cabero, 2010), pues su uso potencializa que el estudiante disfrute su aprendizaje y por lo tanto incremente su calidad durante este proceso. La intención no es aplicar por entretener a los estudiantes, estas mediaciones deben ser diseñadas cuidadosamente para que logren su propósito, de otra forma solo serán simples juegos para pasar un buen rato e incluso podría perjudicar la calidad educativa.

Por consiguiente, la gamificación, entendida como el uso de juegos para aprender, este caso una segunda lengua y los temas fundamentales del inglés, se concentra en la creación de software que les van dando puntos, escudos, insignias, pódiums, similares a los videojuegos, sin que dichos estímulos tengan significado directo con las notas de clase, para no caer en la motivación extrínseca. 
De tal forma que los docentes con una consciencia clara sobre su papel en el aula podrán encaminar los procesos de enseñanza aprendizaje por una generación de un ambiente de motivación permanente. Son útiles, en esta tarea los postulados de varios teóricos como Deci y Ryan (2002), creadores de la Teoría de la Autodeterminación y Perkins (1992) y Schunk (2012); pero especialmente de Keller (2000) quien es el autor de la Teoría Motivacional ARCS (Atención, Relevancia, Confianza y Satisfacción). Cada término de la sigla corresponde a una parte de la clase donde se aplicarán las mediaciones TIC y se analizará el grado de motivación que alcanzan los estudiantes durante los diferentes procesos.

A partir de estas reflexiones, se planteó el objetivo de diseñar una serie de secuencias didácticas gamificadas para la enseñanza del inglés, con estudiantes de $7^{\circ}$ grado (básica secundaria) bajo los lineamientos de los procesos motivacionales en este campo del saber. Para ello, debió identificarse usos y herramientas TIC que puedan incorporarse en este proceso, para mejorar, tanto los niveles motivacionales del estudiantado, como el aprendizaje significativo de inglés. Con ello, el diseño guiado por las TIC, estructurado en un plan de aula se organizó para cada una de las clases con el fin de que se apliquen mediaciones motivacionales de tal manera que los estudiantes, de principio a fin, estén en un ambiente de motivación permanente.

El diseño de secuencias didácticas encadenadas, con el apoyo de vídeo juegos y un plan de acción ampliado, permitirá llevar adelante una investigación mayor bajo el método de Investigación Acción. En esta oportunidad se presenta la organización $\mathrm{y}$ producción de las actividades diseñadas, especialmente, para un grupo de estudiantes a quienes se pretende incorporar a unas dinámicas interactivas en el aprendizaje del inglés mediante el uso de las TIC.

\section{MÉTODO}

El enfoque de investigación fue cualitativo y el diseño el de Proyecto de Aula bajo un plan estructurado de secuencias didácticas que completarán a posteriori un proceso general de Investigación Acción Educativa. E1 trabajo metodológico con proyectos de aula se soportó sobre bases estratégicas en donde tanto los docentes como los estudiantes se convierten en sujetos activos en un trabajo integrado (Perilla y Rodríguez, 2014) que parte de la movilización continua entre teoría y práctica.

Monje (2011) explica que la investigación cualitativa se fundamenta en la hermenéutica, la fenomenología y el interaccionismo simbólico; de la hermenéutica se trata de un estudio con 
seres que piensan, son reflexivos, se comunican e interactúan entre sí, quienes desarrollan subjetividades, toman decisiones y por esto son libres y autónomos, lo que hace que la investigación se mueva más en significados que en datos; se preocupa en comprender los significados de los fenómenos que no solamente explicarlos en términos de causalidad; se tienen en cuenta las intenciones, las motivaciones, las razones, las esperanzas de los estudiantes, más a las prácticas que a los hechos.

La fenomenología interpreta al mundo a partir del sentido que adquieren las cosas para las personas para la creación de su mundo, de su proyecto de vida, de la significación en sus existencias; así, son muy importantes las vivencias de los estudiantes para la presente investigación. El interaccionismo simbólico expone que la conducta humana se puede comprender a partir de las acciones que las personas hacen con las cosas y la significación que dicho evento marca para ellas.

La investigación cualitativa no se preocupa tanto del resultado de la relación de las variables, sino en atrapar la realidad social a través de la percepción de la gente involucrada en el evento, el investigador conceptualiza sobre la realidad basado en el comportamiento, los nuevos conocimientos, las actitudes y valores, este tipo de investigación toma los datos observados y los compara con los comportamientos antes de hacer interpretaciones de los estudiantes observados.

En cuanto a la IAE, La Torre (2007) en $\mathrm{su}$ recopilación de definiciones sintetiza la investigación-accióneducativa como una descripción de una familia de actividades que realizan los docentes en clase y fuera de ellas con fines tales como el desarrollo curricular, su autodesarrollo profesional, la mejora de los programas educativos, los sistemas de planificación, la política de desarrollo y el impacto que tienen sus prácticas en el mejoramiento de sus estudiantes.

La Torre (2007) afirma que "se considera como un instrumento que genera cambio social y conocimiento educativo sobre la realidad social y/o educativa, proporciona autonomía y da poder a quienes la realizan" (p. 23). En este caso el tema a tratar es de carácter pedagógico, con aplicaciones didácticas y énfasis en lo sicológico debido a la importancia de la motivación dentro de las aplicaciones. De acuerdo con Kemmis y McTaggart (1988) en la investigación-acción-educativa las personas trabajan con la intención de mejorar sus propias prácticas:

la investigación debe seguir una espiral que mira los sentimientos y la conciencia de cada uno: una espiral de ciclos de planificación, acción, observación y reflexión; es un todo un sistema de autoaprendizaje, orientado a la praxis (acción críticamente informada y comprometida). (s.n.) 
La Torre (2007) insiste en que la Investigación Acción "empieza con pequeños ciclos de planificación, acción, observación reflexión, avanzando hacia problemas de más envergadura; la inician pequeños grupos de colaboradores, expandiéndose gradualmente a un número mayor de personas" (p. 25). Asimismo, todo lo que se considera como teoría debería ser llevado a la práctica, el investigador al estar presente se hace parte de las prácticas que está investigando; es un actor, y ese mismo investigador es el que hace la reflexión de lo que ocurre durante la investigación, del diario acontecer, de la forma en que se va transformando el quehacer pedagógico dentro de su labor docente investigada por él mismo.

Niño (2011), a partir de Lewin, expresa que la Investigación Acción sigue unos pasos que son: "planificar, actuar, observar y reflexionar" (p. 37). Por lo tanto, se debe planificar, utilizar las técnicas de recolección de datos a través de la entrevista, la encuesta (personal, o grupal) y luego incluir la reflexión, el diálogo y análisis permanente de lo vivido durante la aplicación de los diseños motivacionales y sus consecuencias cuya introspección será permanente durante el proceso, cuyas conclusiones serán mejoradas y aplicadas de nuevo buscando mejorar las prácticas educativas.

\section{Población y sujetos de la investigación}

$\mathrm{La}$ población pertenece a la Institución Educativa Agustín Nieto Caballero, Cali, Colombia, y los sujetos fueron los 32 estudiantes del grupo 7-1 de la sede central, jornada de la mañana, en donde hay algunos jóvenes que llegan de otras instituciones educativas, o sedes de la misma institución, con problemas particulares. Muchos de ellos registran solicitudes de cambio de ambiente educativo por los comités de convivencia de sus planteles; también existen estudiantes que reprobaron el curso en el año inmediatamente anterior o hasta 2 anteriores. Igualmente, están inscritos estudiantes en extra edad, es decir con varios años cronológicos por encima del grupo etario de sus compañeros (por ejemplo, con 16 años en el sexto curso, en donde sus condiscípulos tienen entre 10 y 12 años).

Es una práctica común que esta amalgama de circunstancias negativas se repita en un solo curso, afectando el normal desenvolvimiento del trabajo académico y disciplinario. Por consiguiente, la mayoría de los padres o acudientes de estos jóvenes, están acostumbrados a que sean llamados permanentemente por los profesores $\mathrm{o}$ coordinador de convivencia y ya no se alarman por los desmanes o poca motivación de sus hijos. Normalmente, se espera que en un grupo como este, la convivencia no sea la óptima para el aprendizaje, y como consecuencia el rendimiento académico no sea el mejor; 
no es extraño que al final de año lectivo el índice de morbilidad académico y la deserción sean altos.

Para trabajar con estos sujetos, se utilizan dos tipos de muestreo: el de conveniencia (también llamado de voluntarios) y el teórico (o intencionado) (Monje, 2011). Inicialmente, se utiliza el primero, donde participarán en las encuestas o conversaciones aquellos que deseen hacerlo activamente y de manera reflexiva sobre las experiencias vistas en el aula; luego de detectar aquellos estudiantes y padres de familia que den la información más valiosa sobre las sensaciones vividas durante la aplicación de los diseños motivacionales se concentrará el trabajo futuro en ellos, pues son la representatividad sin que sea la generalidad del grupo, pues en las investigaciones cualitativas se busca extrapolar un resultado, induciendo una teoría que pueda ser usada en otros casos.

La estrategia con este grupo representativo fue indagar con muchas conversaciones y entrevistas, hasta que ocurra la saturación de información y esta comience a ser redundante, para ello se utilizaron los procesos de codificación abierta y codificación axial referidos por Strauss y Corbin (2002) y señalados por Rojas de Escalona (2010) como recursos para el procesamiento y análisis de la información. Esta forma de codificar permitió revisar los contenidos de las entrevistas hasta crear ordenaciones suficientemente útiles para precisar categorías centrales de los fenómenos que se irán encontrando a cada paso, en la investigación mayor.

A partir de toda esta clasificación y codificación se fue decantando la población hasta encontrar los sujetos representativos o cuya información aporte mucho al tema investigado. "Se pretende a través de la elaboración de ejes o tipologías discursivas, la representación socio-estructural de los sentidos circulantes en un determinado universo $\mathrm{y}$ con relación al tema a investigar" (Monje, 2011, p. 130). Los fundamentales fueron los casos extremos, aquellos estudiantes que no se destacan, que en ocasiones han demostrado el poco gusto por la academia, descartando los típicos o representativos de la comunidad en general.

\section{Técnicas e instrumentos de recolección de información}

Una de las formas de volver sobre la actitud y desarrollo del trabajo de los estudiantes en este Proyecto de Aula fue la observación de videos sobre su participación y su grado de entusiasmo durante las clases.

Durante la aplicación de la secuencia didáctica y la ejecución del proyecto se utilizó la observación participante, pues el investigador tiene una relación educativa y es conocido y conoce a los sujetos investigados e irá describiendo lo que observó durante la 
clase en que se aplicó el diseño motivacional. Estas anotaciones son clasificadas, de acuerdo con Hernández Sampieri, Fernández y Baptista (2010) en: (a) directas donde se narran de los hechos ocurridos, en este caso la grabación de los videos de las clases y escribir lo observado; (b) interpretativas, con comentarios sobre los hechos, lo que se sintió, las experiencias nuevas, lo que llamó la atención, lo inesperado; (c) temáticas, dedicadas a manifestar ideas, hipótesis, preguntas de investigación, especulaciones relacionadas con la teoría, conclusiones preliminares que van arrojando las observaciones. Para esto se debe revisar cada cierto tiempo, por ejemplo, cada 3 clases todo el material y hacer comparaciones que pueden brindar todo ese material.

También se realizaron anotaciones. Según Hernández Sampieri, Fernández y Baptista (2010) de tipo personales que incluyen los sentimientos, las sensaciones del investigador, lo que se piensa sobre la investigación, si ya se está llegando al estado de saturación; anotaciones de la reactividad de los participantes que incluyen los cambios producidos por el investigador sobre los participantes: es importante que el investigador escoja los sujetos realmente representativos del grupo, los que le den mejor información, los que tengan mejores sentimientos hacia el trabajo investigado o lo contrario, si esto fuese útil, los que peor se sienten en el trabajo.
Fue oportuno tener en cuenta, además, la lista de objetos que intervienen en la investigación, como artefactos electrónicos de la institución, de los estudiantes, del apoyo a la investigación, de los elementos de los estudiantes que llevan diariamente a clase como cuadernos, textos, smartphones, consolas portátiles de videojuegos, raspberries, simuladores de gafas VR y hasta simuladores de videojuegos para computadores.

Asimismo, para este tipo de trabajos con videos y similares se recolectaron los permisos de consentimiento informado para realizar las actividades, previamente descritas, por parte de los padres de familia de los participantes de la investigación y las entidades pertinentes.

E1 propio software generado para la investigación ofreció a los participantes, al final de cada juego, una encuesta. Estas encuestas contienen preguntas de respuestas cerradas, pero con una gradualidad que indican la intensidad de la respuesta, donde 1 es muy pobre y 10 es superó las expectativas.

Además, se realizaron una serie de entrevistas dirigidas (Monje, 2011) a algunos estudiantes del muestreo de voluntarios y otras también dirigidas y en profundidad, focalizadas al tema investigado. Finalmente, luego de aplicadas las secuencias y avanzados los proyectos se llevó a cabo el mismo tipo 
de entrevistas a los padres, para confrontar lo observado por el investigador y lo que manifiestan los estudiantes sobre la clase y el software.

\section{RESULTADOS Y DISCUSIÓN}

En esta primera fase del trabajo, propia de la implantación de Proyecto de Aula, se llevó a cabo la creación de una aplicación gamificada (Ropero, 2018), la cual se implementó en el curso 7-1 2020 con 38 estudiantes. E1 diagnóstico inicial indicó que se presenta un bajo rendimiento académico general y desmotivación para el aprendizaje, y también se reportaron algunas conductas que interfieren con el respeto y atención debida a las clases.

Seguidamente, se seleccionaron otras aplicaciones que complementaron las mediaciones TIC, además de la aplicación creada. Y se realizaron revisiones teóricas, actividades prácticas y procesos evaluativos que le dieron continuidad a las secuencias didácticas.

\section{El producto de la propuesta}

Antes de las muestras aquí desplegadas, se realizaron algunas pruebas piloto, con las que se logró probar algunos diseños motivacionales con buena recepción por parte de estudiantes y padres de familia. Pese a la falta de elementos básicos para una clase virtual, los primeros videos grabados y las entrevistas efectuadas nos dan mucha información que permite desarrollar, en el futuro, la simbología apropiada para usar durante el registro en este tipo de investigación. En medio de estas fases, estuvo en proceso la creación y aplicación del video juego "Desafío San Buenaventura" el cual fue diseñado por el estudiante de sistemas Nelson Zambrano y el profesor Edgar Ramírez Ramírez, autor de este trabajo. (Figura 1) El vídeo juego ha sido creado en la plataforma Unity versión 2018.4.15fs; inicialmente, se comenzó a trabajar en red con computadores, en la institución, pero debido a la emergencia se le están haciendo modificaciones para trabajar de manera online. (Se presentan algunos ejemplos de la interfaz). 

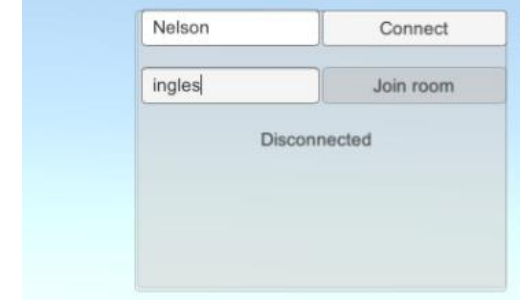

(1)

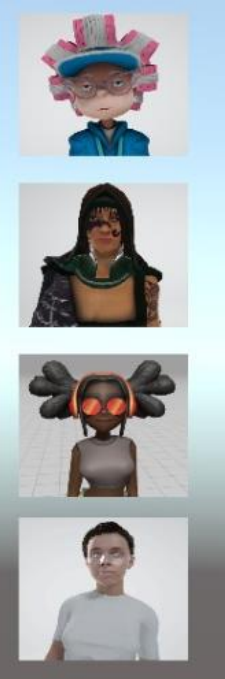

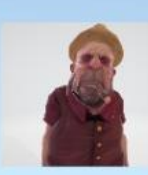
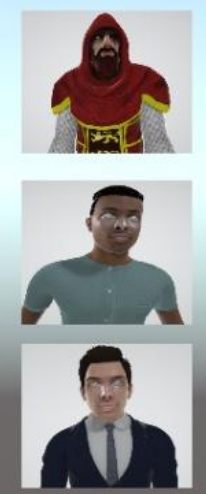
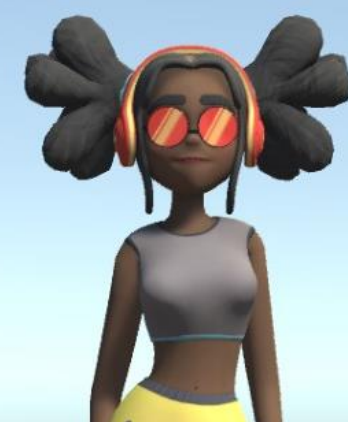

El estudiante descarga desde un correo un software que lo conecta antes de la sesión que consiste en una competencia donde pueden ingresar hasta 50 jugadores a pasar un laberinto el cual cuenta con 10 pasos equivalentes a 10 preguntas sobre el tema que el profesor desee evaluar (Figura 2). Es muy útil, en especial con preguntas de comprensión lectora de selección múltiple, con una o varias respuestas verdaderas, las cuales se pueden responder después de una lectura o, para aumentar la presión de la competencia, se pueden hacer al mismo tiempo que la lectura. El texto completo, de manera física, está en manos del estudiante, pero también puede ver parte de él durante la exigencia o no de acuerdo con la programación efectuada por el docente. 


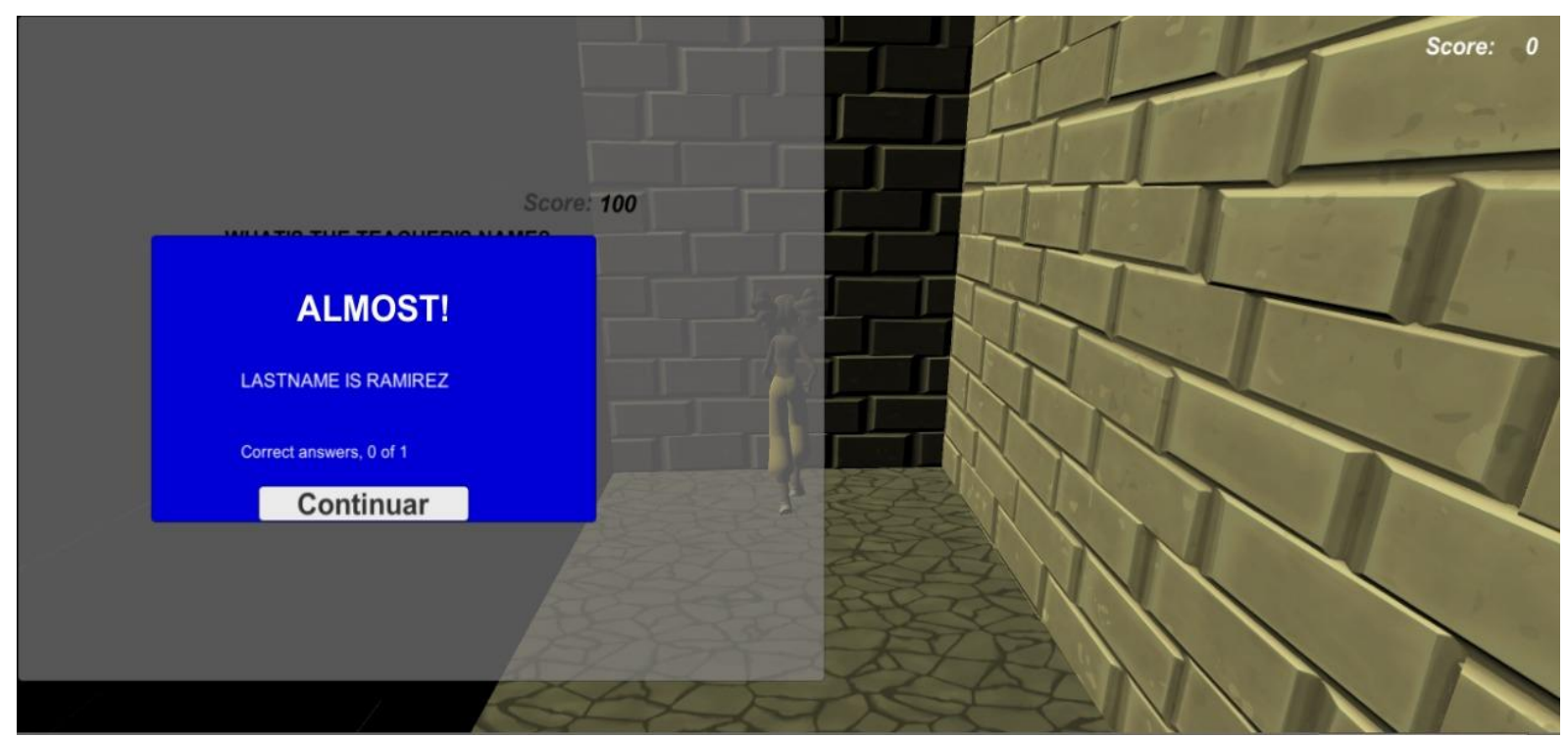

Figura 2. Pregunta no acertada, se evita el uso de la expresión “Try Again”. (Fuente: Elaborado por el autor)

Se puede programar el tiempo de juego y la cantidad de jugadores, igualmente es posible programar el tiempo de respuesta en cada pregunta: si el jugador no responde acertadamente $o$ se le termina el tiempo, es regresado al punto anterior y en el nuevo desafío aparecerá una pregunta diferente, pero la fallada volverá a presentarse más adelante. Una vez terminado el tiempo del juego, entre 5 y 10 minutos -o más si desea el docente-, se terminará el juego y surgirá un ranking que indicará la posición, nombre, preguntas acertadas, $\mathrm{y}$ tiempo, teniendo como primera variante las preguntas contestadas correctamente y luego el tiempo de llegada. Sobre esta actividad, ya se han hecho 3 pruebas piloto, con otros cursos, con resultados que demuestran que el juego gusta mucho a los estudiantes y puede ser valioso en la investigación.
La plataforma mostrará un laberinto diferente en cada juego (Figura 3), de manera que no se vuelva monótono para el estudiante, también se pueden asignar audios ambientales en cada juego, para que así en una partida tengan música electrónica, reggaetón, salsa o cumbia. Una de las fortalezas sobresalientes de este juego es que se puede realizar en intranet cuando no se cuente con Internet, para lo cual se debe instalar en cada uno de los equipos y conectarse en red con otro computador que hace de servidor. También puede ser jugado en línea mediante una red LAN; igualmente se puede hacer un desarrollo posterior que lo convierta en una aplicación móvil para jugar desde los smartphones, mediante una migración APK. 


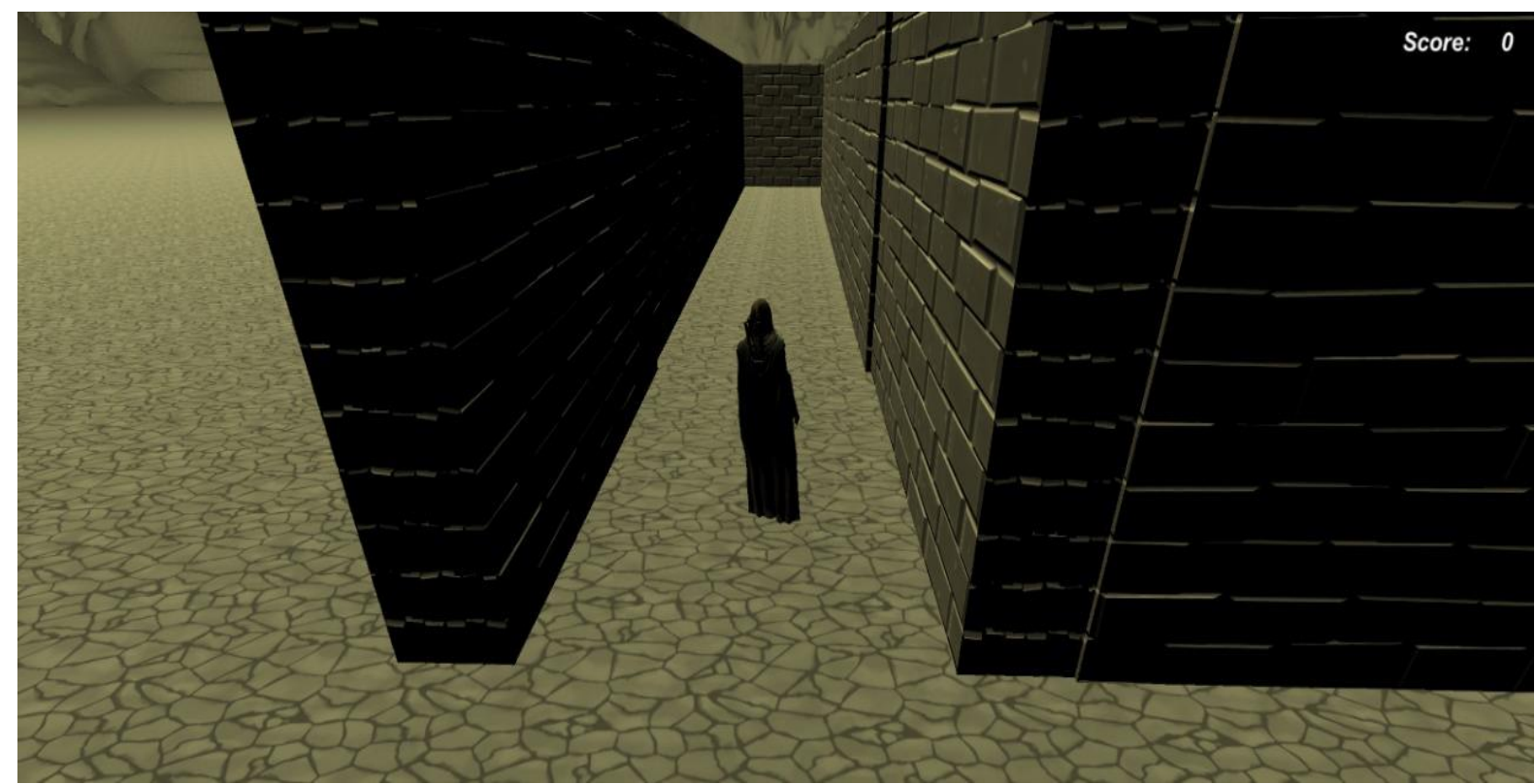

Figura 3. Escenario de la búsqueda del acertijo. (Fuente: Elaborado por el autor)

Para medir la motivación del estudiante (Figura 4), el juego tiene al final la opción de realizar encuestas anónimas, las cuales equivalen a las herramientas motivacionales propuestas por Pintrich y Schunk. (2006)

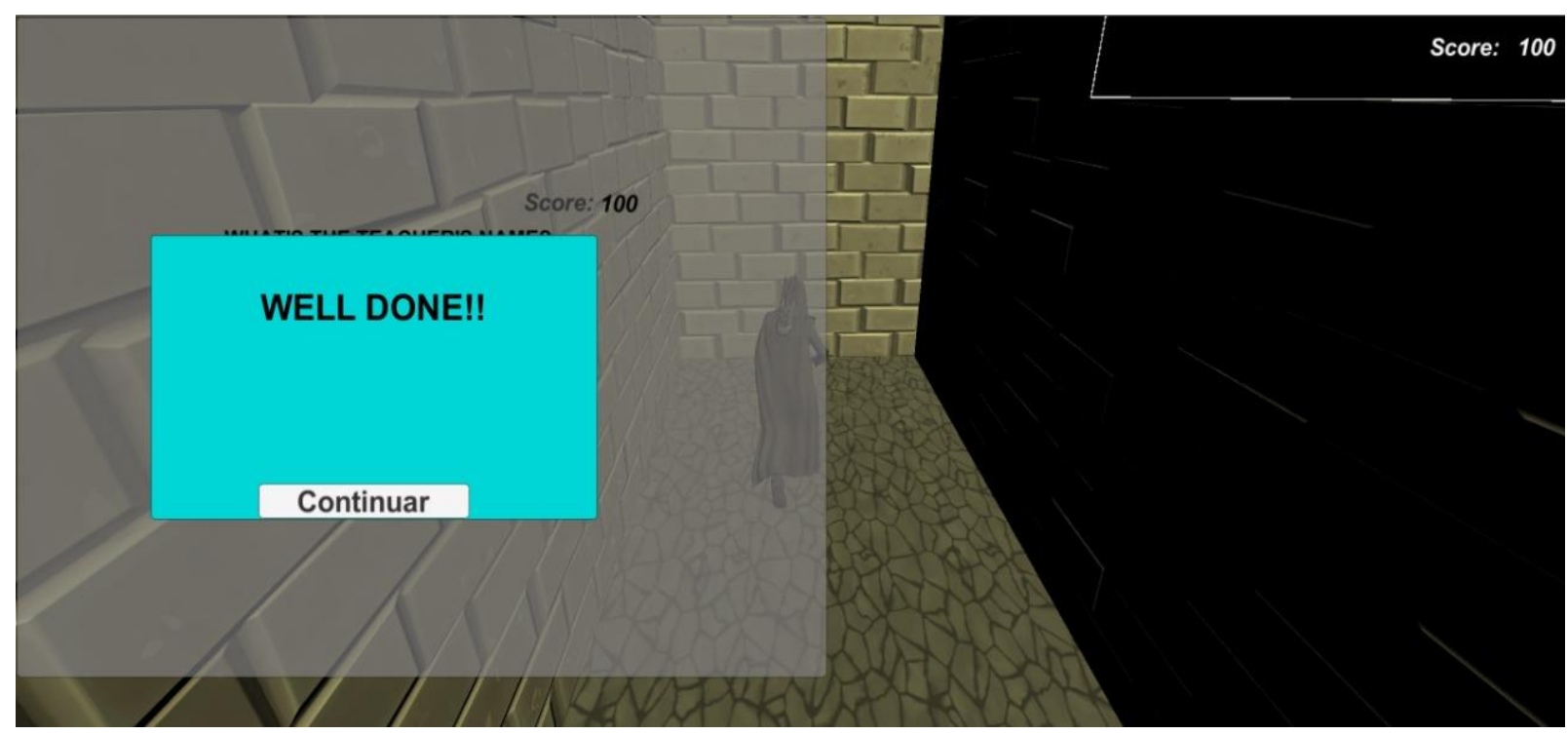

Figura 4. Respuesta correcta. (Fuente: Elaborado por el autor) 
Además de ese software, durante las clases se usarán otras mediaciones que complementarán el trabajo de la aplicación creada tales como:

1. Edmodo (LMS), la cual es una plataforma gamificada donde se envían, reciben, programan y se hacen exámenes en línea, se mantiene comunicación permanente con los estudiantes en un ambiente similar a Facebook, de allí que se le conozca como una red social educativa; funciona tanto desde celular, PC o tableta.

2. Plickers es una aplicación relacionada con realidad aumentada, la cual usa un computador, un proyector, conexión de internet que pueden ser datos, y una serie de tarjetas prestablecidas que corresponden a las respuestas de selección múltiple que son escaneadas por un smartphone y de esa forma realiza evaluaciones instantáneas.

3. Kahoot es una aplicación que utiliza un computador y proyector con los que trabaja el profesor y varios computadores, tabletas o smartphones con los que juegan los estudiantes sobre un tema propuesto por el profesor, es un desafío que muestra un pódium momentáneo de los jugadores y produce mucho entusiasmo entre los estudiantes.

4. Quizizz es una aplicación similar a Kahoot, pero no es necesario el proyector. Estas dos últimas herramientas también tienen una posibilidad de trabajar a distancia, donde el profesor hace el desafio con un tema, los estudiantes entran muchas veces a resolver las preguntas y cada vez que entran y mejoran, tienen un puntaje que se refleja en una tabla que le da la aplicación al docente. Muy útil para repasar mientras los estudiantes lo implementan en un estilo videojuego.

5. Google Docs es una mediación evolucionada de los Wikis, en la cual varias personas trabajan en un solo documento, que debe ser construido de manera cooperativa.

6. Google Photos es una aplicación con la cual se toma una foto del cuaderno donde hizo el trabajo de clase el estudiante, y de manera inmediata pasa al computador proyectándolo a la clase, eliminando el uso de cartulinas o pliegos de papel grandes usados tradicionalmente para hacer las exposiciones.

En cada revisión, activación y aplicación los estudiantes se sienten motivados y continúan trabajando $\mathrm{y}$ reportando sus experiencias. Así como se sigue tomando notas sobre su proceso de aprendizaje.

Sobre el éxito en este tipo de actividades educativas deben tomarse en cuenta las virtudes ya mencionadas en la teoría y en diversos estudios previos. En un trabajo realizado por Escobar (2016) se diseñó y llevó a cabo la aplicación de una propuesta asistida por el material hipermedial destinada a docentes para su uso en la enseñanza del idioma 
inglés. En esta investigación se mostró que los materiales de las TIC brindan medios útiles para los profesores debido a que pueden incrementar sus competencias para producir estrategias, tareas y actividades. Asimismo, se relacionó con la motivación para alcanzar los propósitos de la asignatura en cuanto que al tener una intención sabida y divulgada entre los alumnos y docentes se favorecen los procesos de enseñanza y aprendizaje.

A partir de experiencias como esta se puede considerar que los recursos tecnológicos integrados con un diálogo abierto y un objetivo conocido por todos estimula las motivaciones y la autonomía de aprendizaje. También, se accede a la posibilidad de que los estudiantes continúen construyendo por sí solos luego de tener estas experiencias. Esa continuidad ofrece un sentido distinto a la propia configuración de la actividad de aula y la multiplica para brindar nuevos conocimientos, en el idioma inglés, tanto conceptuales como procedimentales.

Todas las aplicaciones informáticas podrían convertirse en recursos constantes para la educación con la finalidad de adquirir la habilidad de un segundo idioma, con el agregado de que los diversos elementos del software han mejorado los desarrollos de las cuatro habilidades: hablar, escuchar, leer y escribir (Kalaiarasan y Somasundaram, 2015). Las deficiencias, como vocabulario, gramática, patrones de habla y pronunciación pueden seguir mejorando con un grupo motivado a través de juegos planificados. Los trabajos mediante las TIC para desarrollar las habilidades en inglés son, en sí mismos, los propios vehículos de lenguaje ya que movilizan las destrezas de los alumnos de manera efectiva y exitosa mediante una variedad de actividades.

A través de las TIC se utilizan una diversidad de métodos de capacitación, ejercicios y prácticas para lograr la competencia de los alumnos en la aplicación del conocimiento. Las cuatro habilidades se internalizan con una experiencia de aprendizaje agradable, generan un entorno de aprendizaje significativo y promueven el pensamiento de orden superior (Zohar, 2006). Al usar las aplicaciones, los alumnos pueden controlar el tiempo y la duración del estudio requerido para el dominio de los contenidos; sus esfuerzos les garantizan puntos y victorias por lo que siempre se sienten ganadores y el aprendizaje será mucho más rápido.

\section{CONCLUSIONES}

El impacto emocional de las prácticas educativas planificadas en los jóvenes estudiantes es un tema que debe seguir profundizándose a pesar de la existencia de la variedad de estudios. Se puede liderar el reforzamiento de dichas actividades, o llegar a favorecer verdaderos cambios con aquellas que produzcan nuevas sensaciones positivas. En este caso los estudiantes se sintieron 
entusiasmados con la clase $y$ se dedicaron a participar con el vídeo juego creado especialmente para ellos.

El diseño de la clase, utilizando mediaciones TIC, es lo que hace la diferencia entre el aprendizaje basado en juegos, con el manejo de la tecnología solo por entretenimiento; y si en ese diseño dichas mediaciones TIC tienden a crear y sostener el entusiasmo durante toda la actividad académica, los estudiantes disfrutan la experiencia y el aprendizaje está asegurado.

Finalmente, hay que acotar que se sigue perfeccionando el software $y$ mejorando cada interfaz en la medida en que los estudiantes continúen trabajando desde sus dispositivos electrónicos y PC. La propuesta servirá como motivación y mejora en su conocimiento del inglés y podrán ver sus propios avances.

\section{REFERENCIAS}

Anaya, Y., Díaz, S., y Martínez, J. (2012). El uso de las TIC como herramienta para el aprendizaje significativo del inglés. Revista Rastros Rostros, 14(27), 115-119

Cabero, J. (2010). Los retos de la integración de las TICs en los procesos educativos. Límites $\mathrm{y}$ posibilidades. Revista Perspectiva Educacional, $\quad 49(1), \quad 32-61$. Recuperado de http://www.perspectiva educacional.cl/index.php/peducaci onal/article/view/3
Camposeco, F. de M. (2012). La autoeficacia como variable en la motivación intrínseca $y$ extrínseca en matemáticas a través de un criterio étnico. (Tesis de doctorado). Universidad Complutense de Madrid, España. Recuperado de https://eprints.ucm.es/16670/1/T3 4002.pdf

Deci, E. y Ryan, R. (2002). Handbook of Self-determination Research. Rochester, NY: University of Rochester Press

Escobar, F. (2016). El uso de las TIC como herramienta pedagógica para la motivación de los docentes en el proceso de aprendizaje $y$ enseñanza en la asignatura de inglés. (Trabajo de Maestría), Universidad Pontificia Bolivariana, Medellín, Colombia. Recuperado de https://repository. upb.edu.co

/bitstream/handle/20.500.11912/2 762/TESIS_MTIC_FLOR_ESCO BAR $\% 20-\% 20 F I N A L \% 20 \% 281 \%$ 29.pdf?sequence $=1$

Hernández Sampieri, R., Fernández, C. y Baptista, P., (2010). Metodología de la investigación.( $5^{\mathrm{a}}$ ed.). México: McGraw Hill

Kalaiarasan, M. y Somasundaram, G. (2015). Software Applications and Second Language Acquisition. Journal of Technology for ELT. Recuperado de https://sites.google. com/site/journaloftechnologyforelt /archive/october---december2012/4-software-applications-andsecond-language-acquisition

Keller, J. (2000). How to integrate learner motivation planning into lesson planning: The ARCS model approach. U.S.A: Florida State University. 17. Recuperado http://apps.fischlerschool.nova.edu 
/toolbox/instructionalproducts/itde 8005/weeklys/2000-Keller-

ARCSLessonPlanning.pdf

Kemmis, S. y McTaggart, R. (1988). The Action Research Planner. (Third Edition) Melbourne, Australia: Deakin University Press

La Torre, A. (2007). La investigaciónacción. Conocer y cambiar la práctica educativa. Barcelona, España: Editorial Graó

Monje, C. A. (2011). Metodología de la investigación cualitativa y cuantitativa. Guía Didáctica. Neiva, Colombia: Universidad Surcolombiana. Recuperado de https://www.uv.mx/rmipe/files/2 017/02/Guia-didacticametodologia-de-la-investigacion.pdf Niño, V. (2011). Metodología de la investigación, diseño y ejecución. Bogotá, Colombia: Ediciones de la U. Recuperado de http://roa.ult.edu.cu/bitstream/12 3456789/3243/1/METODOLOGI A\%20DE\%20LA\%20INVESTIGA CION\%20DISENO\%20Y\%20EJE CUCION.pdf

Orbegoso, A. (2016). La motivación intrínseca según Ryan y Deci y algunas recomendaciones para maestros. Educare, Revista Cientifica de Educação. 2(1), 75-93. Recuperado de DOI: http://dx.doi.org/10.19141/24475432/lumen.v2.n1.p.75-93

Perilla, L. y Rodríguez, E. (2014). Proyectos de aula: una estrategia didáctica hacia el desarrollo de competencias investigativas. Revista Episteme, 6-7. Recuperado de https://docplayer.es/37874222Proyectos-de-aula-una-estrategiadidactica-hacia-el-desarrollo-decompetencias-investigativas-por- lucila-perilla-ruiz-y-2-elsa-rodriguezpaez.html

Perkins, D. (1992). La escuela inteligente. USA: Free Press Print, Universidad de Harvard

Pintrich, P. R. y Schunk, D. H. (2006). Motivación en contextos educativos. Teoría, investigación y aplicaciones. $\left(2^{\circ}\right.$ ed.). Madrid: Pearson Education

Rojas de Escalona, B. (2010). Investigación cualitativa. Fundamentos $y$ praxis. Caracas: FEDUPEL

Ropero, S. (2018). Aplicación de una metodología gamificada para la mejora de una Unidad Didáctica en Formación Profesional. (Trabajo de Maestría) Universidad Internacional de La Rioja, España. Recuperado de https://reunir.unir.net/bitstream/h andle/123456789/7020/ROPERO \%20MORALES\%2C\%20SONIA.p $\mathrm{df}$ ? sequence $=1$ \&isAllowed $=\mathrm{y}$

Santos Marín, U. (2017). La motivación de elección para aprender español, inglés y otras lenguas en Europa del Norte: el caso de Estonia. (Tesis de doctorado). Universidad de Granada, España. Recuperado de https://digibug.ugr.es/handle/1048 $1 / 48123$

Schunk, D. (2012). Learning Theories an Educational Perspective. Sixth Edition. USA: Pearson Printer. Recuperado de

https://www.researchgate.net/file. PostFileLoader.htmlid $=53 \mathrm{ad} 2847 \mathrm{c}$ f57d75c068b45c5\&assetKey $=$ AS $\% 3$ A273549456019456\%401442230680 395

Strauss, A. y Corbin, J. (2002). Bases de la investigación cualitativa. Técnicas y procedimientos para 
desarrollar la teoría fundamentada. Antioquia, Colombia: Editorial Universidad de Antioquia

Vivar, C., Arantzamendi, M., LópezDicastillo, O. y Gordo, C. (2010). La Teoría Fundamentada como Metodología de Investigación Cualitativa en Enfermería. Index de Enfermería, 19(4), 283-288. Recuperado de http://scielo.isciii .es/scielo.php?script=sci_arttext\&pi $\mathrm{d}=$ S1132-12962010000300011\&lng $=$ es\&tlng $=$ es
Zohar, A. (2006). El pensamiento de orden superior en las clases de ciencias: objetivos, medios y resultados de investigación. Enseñanza de las ciencias, 24(2), 157172. Recuperado de https://www.researchgate.net/publ ication/39383420_E1_pensamiento_ de_orden_superior_en_las_clases_d e_ciencias_objetivos_medios_y_res ultados_de_investigacion 